\title{
1 Serine/Arginine-rich protein family of splicing regulators: New approaches to study 2 splice isoform functions
}

4 Mitchell Morton ${ }^{1,4}$, Nadia AlTamimi ${ }^{1,4}$, Haroon Butt ${ }^{2,4}$, Anireddy S.N. Reddy ${ }^{3}$ and Magdy

5 Mahfouz $^{1,2, *}$

7 Division of Biological Sciences, 4700 King Abdullah University of Science and Technology, Thuwal

8 23955-6900, Saudi Arabial

9 Laboratory for Genome Engineering, Division of Biological Sciences, 4700 King Abdullah University of

10 Science and Technology, Thuwal 23955-6900, Saudi Arabia ${ }^{2}$

11 Department of Biology, Program in Cell and Molecular Biology, Colorado State University, Fort Collins, $12 C O, U S A^{3}$.

13

14 These authors contributed equally to this work ${ }^{4}$

$15 *$ *Correspondence: magdy.mahfouz@kaust.edu.sa

\section{Keywords}

17 Splicing, Alternative Splicing, SR proteins, RNA-binding Proteins, Splice variants, Genome 18 engineering, Stress

19 Highlights:

20 - Serine/arginine-rich (SR) proteins bind RNA and function in stress responses

21 - Conserved SR proteins modulate alternative splicing and other aspects of RNA 22 metabolism

- New technologies (CRISPR/Cas, TRIBE) enable functional analysis of SR families and their splice isoforms 


\section{Abstract}

Serine/arginine-rich (SR) proteins are conserved RNA-binding proteins that play major roles in RNA metabolism. They function as molecular adaptors, facilitate spliceosome assembly and modulate constitutive and alternative splicing of pre-mRNAs. Pre-mRNAs encoding SR proteins and many other proteins involved in stress responses are extensively alternatively spliced in response to diverse stresses. Hence, it is proposed that stress-induced changes in splice isoforms contribute to the adaptation of plants to stress responses. However, functions of most $S R$ genes and their splice isoforms in stress responses are not known. Lack of easy and robust tools hindered the progress in this area. Emerging technologies such as CRISPR/Cas9 will facilitate studies of $S R$ function by enabling the generation of single and multiple knock-out mutants of SR subfamily members. Moreover, CRISPR/Cas13 allows targeted manipulation of splice isoforms from SR and other genes in a constitutive or tissue-specific manner to evaluate functions of individual splice variants. Identification of the in vivo targets of SR proteins and their splice variants using the recently developed TRIBE (Targets of RNA-binding proteins Identified By Editing) and other methods will help unravel their mode of action and splicing regulatory elements under various conditions. These new approaches are expected to provide significant new insights into the roles

Introduction

RNA binding proteins (RBPs) play essential roles in every aspect of RNA metabolism from biogenesis, pre-mRNA splicing and polyadenylation to RNA modification, transport, localization, stability, and translation, ultimately dictating the quantity and functions of RNAs and the proteins they encode. Moreover, modulation of RNA metabolism by RBPs has important regulatory roles in plant development and environmental responses. Here, we examine the role of the conserved serine-arginine (SR) family RBPs in post-transcriptional gene regulation, development, and stress responses in plants. We also discuss the utility of emerging technologies aimed at targeted gene editing, targeted mRNA degradation and identification of in vivo targets of SRs in understanding 53 54 55 


\section{SR Proteins in Plants}

The SR proteins containing the arginine- and serine-rich (RS) domain were discovered nearly 30 years ago in studies that linked RS domain-containing proteins and splicing regulation [1]. These were among the first regulators of pre-mRNA splicing, identified through genetic analyses in Drosophila [2]. Animal SR proteins possess one or two N-terminal RNA recognition motifs (RRMs), which determine RNA-binding properties, and a reversibly phosphorylated Cterminal RS domain, that facilitates protein-protein interactions [3]. Plant SR proteins are defined as having one or two N-terminal RRM and a C-terminal RS domain of at least 50 amino acids (aa) with a minimum SR or RS dipeptide content of $20 \%$, as opposed to $40 \%$ in Metazoa [3]. Bioinformatic analyses have revealed that plants in general, and angiosperms in particular, possess the most diverse set of SR proteins of all eukaryotes [4] (Figure 1). For example, Arabidopsis thaliana has 18 SR genes, Oryza sativa has 22 but Caenorhabditis elegans has only 7 and humans have $12[3,5,6]$. Plant SR proteins are categorized into six subfamilies [3], three of which (SR, RSZ, and SC subfamilies) are orthologous to animal SR proteins, while the other three are plantspecific and have structural features that are not observed in the animal kingdom (Figure 1). For example, members of the SCL subfamily of SR proteins, while structurally related to the SC subfamily, possess a unique N-terminal domain rich in charged amino acids. RS2Z members resemble the RSZ subfamily, yet have an additional zinc knuckle and a serine and proline-rich acidic carboxy-terminal domain [3]. Some studies suggest functional redundancy of SR proteins [7] but the extent to which SR proteins are functionally diversified or redundant is still not completely understood.

Although some studies indicate the conservation of splicing regulatory pathways among higher eukaryotes [8,9], plant introns are imprecisely spliced in mammalian splicing extracts and animal pre-mRNAs are not spliced in plant nuclei, indicating the existence of kingdom-specific splicing regulatory mechanisms. These discrepancies are likely related to structural differences between plant and animal introns, with plant introns tending to be much shorter with a higher $\mathrm{U}$ content $[5,10,11]$. These differences also may provide an explanation for the expansion of the SR protein family in plants [5]. Furthermore, the identification of several plant-specific SR protein subfamilies is indicative of functional diversification, potentially conferring plants additional responsiveness, intricacy, and precision in the regulation of alternative splicing and helping plants to produce the right gene expression patterns in response to growth and stress cues. 


\section{SR proteins regulate constitutive and alternative splicing}

The constitutive splicing (CS) pathway uses consensus $3^{\prime}$ and $5^{\prime}$ splice sites (SS), producing a single mature mRNA that comprises all exons of a given gene. However, alternative splicing (AS) can generate numerous different isoforms from a single pre-mRNA through the selection of alternative splice sites, which can lead to intron retention, exon skipping, the inclusion of cryptic exonic sequences, or various combinations thereof. Alternative splicing is prevalent and indeed essential within higher eukaryotes [12]. In humans, AS is observed in $95 \%$ of multiexon genes and its misregulation is implicated in several severe debilitating diseases [13-15]. In plants, over $60 \%$ of intron-containing genes are alternatively spliced, ranging from $32 \%$ in Oryza sativa $[13,16,17]$ to $61 \%$ in Arabidopsis thaliana $[13,18-20]$. It is expected that the observed prevalence of AS in plants will increase with a more comprehensive analysis of transcriptomes from different plant tissues under different growth and stress conditions, and developmental stages. AS can expand proteome complexity and regulate gene expression through multiple mechanisms. It can produce several mRNA isoforms from a single pre-mRNA, each of which can generate specific protein products with varying structures, thus influencing downstream functions such as binding properties, enzymatic activity and intracellular localization. Additionally, AS is coupled to transcript stability and translation by means of nonsense-mediated decay (NMD) and microRNAmediated gene regulation [21].

The prevalence of AS and the breadth of affected functional gene groups highlight it as a key determinant of plant phenotypes [13]. AS events are extensively regulated and crucially involved in determining cell- and tissue-types in a developmentally regulated manner, driving differentiation in response to various cues through the establishment of context-dependent transcript profiles [22]. Moreover, disproportionately high frequencies of AS in regulatory and stress-related genes $[5,16,20,23,24]$ and the susceptibility of AS profiles to environmentally induced changes support a role for AS in plant adaptation to abiotic stress [5, 19, 25-27].

Whether CS or AS takes place is determined by various cis-acting regulatory sequences and trans-acting RBP splicing factors. Cis elements include consensus sequences such as the 5' and 3' SS, and the branch-point sequence, along with facultative motifs termed exonic/intronic splicing enhancers/silencers (ESE/ESS/ISE/IS), which are collectively called splicing regulatory elements [SREs]) (Figure 2). SREs are recognized and bound by trans-acting splicing factors, such as SR proteins and hnRNPs (heterologous nuclear RNPs) (Figure 2), which can accordingly 
promote or disrupt spliceosome assembly or activity, thus enhancing or suppressing the selection of nearby SS in a concentration-dependent manner [13, 28, 29]. Typically, SR proteins and hnRNPs have antagonistic functions, favoring and opposing splicing in their vicinity, respectively, most frequently by interacting with the U1 and U2 snRNP during spliceosomal assembly [30]. 124 Splicing factors, which are subject to tight spatiotemporal regulation, are responsible for establishing contextual splicing regulation that governs processes ranging from growth and development to responses to environmental stimuli [31]. SR proteins, in particular, have been the

127 focus of substantial interest in recent years for their roles in splicing regulation as well as other aspects of RNA metabolism.

SR proteins bind short, degenerate SREs on cognate pre-mRNAs via the RRM, and occasionally the RS domain can bind 5'SS and branch-point motifs on the pre-mRNA, and subsequently influence spliceosome assembly and splicing through the RS domain [32-35]. SR proteins can also remain bound to spliced transcripts and influence, by means of the RS domain, downstream processes such as mRNA nuclear export, mRNA stability, NMD and translational modulation [3, 31, 36-42]. For instance, the RS domain has been shown to function as a nuclear localization signal, interacting with the nuclear import receptor, transportin-SR, to regulate subcellular localization of SR proteins and, eventually, AS of their target genes. SR proteins are themselves subject to extensive AS that, given their modular domain structure, can lead to substantial changes if either key functional group is excised from the final transcript [43-45]. AS of SR protein pre-mRNAs can also function in controlling their expression through the generation of premature termination codon (PTC+) transcripts that are eliminated via the NMD pathway [46], thereby affecting the expression and splicing of target genes [13]. As such, SR proteins have emerged as major players in splicing and AS but also more generally in RNA metabolism and post-transcriptional gene regulation.

Regulation of SR proteins can be achieved at the transcriptional level, where even minimal 146 nature of interactions with antagonistic splicing factors [13]. SR proteins are also subject to AS 147 themselves, frequently as a result of the activity of other SR proteins, or indeed their own [32, 47]. 148 Finally, SR protein activity can be modulated at the post-translational level by reversible 149 phosphorylation of numerous sites by protein kinases along the RS domain [31, 48]. The nature of 150 the interactions between the highly disordered RS domain and protein partners is heavily 
151 influenced by its phosphorylation state that can generate vast structural rearrangements and thus

152 functional modulation [31]. SR protein kinase phosphorylates SR proteins and controls splicing in 153 both constitutive and alternative splicing [49]. Three mammalian SR protein kinases, including 154 Lammer/CLK kinases, SRPK1, and SPRK2, matched eight homologs in Arabidopsis [50]. 155 Lammer/CLK kinases phosphorylate SR proteins in vitro in plants $[49,51]$. An example of SR protein kinase regulation of AS is the tobacco Lammer/CLK kinase homolog, PK12, that, when overexpressed, changes alternative splicing pattern of U1 small nuclear ribonucleoprotein $70 \mathrm{kDa}$ (U1-70K) and in two SR proteins, atSRp30 and atSR1/atSRp34 pre-mRNAs [49].

\section{SR proteins are implicated in plant responses to stress, growth and developmental signals}

Plant SR proteins are regulated by various developmental and environmental cues, driving context-dependent AS patterns that likely contribute to adaptation to various conditions. Abiotic stresses regulate the expression of SR genes. In Arabidopsis, for example, SR30 is up-regulated in response to salinity and high-light irradiation [45] while SCL33 is down-regulated upon a variety of stresses including salt, heat, and cold, and by abscisic acid treatment [52]. Nevertheless, these stress-induced changes in SR gene expression are observed in relatively few members, and appear to contribute only modestly to stress responses [5].

Stress-induced AS of SR proteins, on the other hand, is widespread, with the AS patterns of numerous SR proteins in Arabidopsis influenced by high-light irradiation, temperature, salinity and hormone application $[25,45,52]$. In particular, AS often leads to the production of PTC+ transcripts that are eliminated via the NMD pathway [44]. In Arabidopsis, AS of SR30 and SR34 is highly light- and heat-sensitive, markedly increasing the concentration of the full-length productive isoform relative to the unproductive PTC+ isoform [25, 45]. As such, stress-induced AS of SR proteins influences stress responses by changing the SR domain structure as well as through changing levels of functional full-length SR protein. Unfortunately, very few functional studies have elucidated the effects of SR AS, or indeed that of the changes in global AS triggered by SR splicing variants.

In addition, SR proteins may be subjected to post-translational regulation in response to abiotic stress through reversible and combinatorial phosphorylation of various phosphosites along the SR domain. These modifications can have various effects, ranging from changes in RNA- 
183 binding and catalytic properties to modulation of subcellular localization. For example, cold184 induced protein phosphorylation drives the redistribution of the noncanonical Arabidopsis SR-like protein SR45 from nuclear speckles to a diffuse nucleoplasmic arrangement [5, 53]. Similarly, localization of RSZ22 in the nucleolus upon prolonged periods of stress is linked to the phosphorylation status of the cell, likely due to depletion of ATP levels $[54,55]$. In fact, ATP levels and phosphorylation have been shown to regulate the mobility of plant SR34 [56].

Alternatively spliced transcripts are implicated in plant growth and development, signal transduction and flowering, with regulatory and stress-responsive genes clearly overrepresented [27, 46, 57-59]. Furthermore, plant SR proteins are regulated by developmental cues at the transcriptional and post-transcriptional levels, indicating that these proteins are targets of several signaling pathways and might act in the response to developmental signals. For example, Arabidopsis plants ectopically expressing a specific SR protein produced elevated protein levels in all tissues [60, 61]. Overexpression of atSRp30, one of the Arabidopsis SF2/ASF homologs, in transgenic plants resulted in developmental changes, exhibited predominantly as a late flowering phenotype, by altering the splicing patterns of various pre-mRNAs [60]. The atSRp30 protein controlled splicing of its own pre-mRNA, as increasing levels of atSRp30 altered the splicing pattern in atSRp34/SR1, another Arabidopsis SF2/ASF homolog. This altered splicing decreased mRNA1, which encodes the full-length protein while greatly increasing mRNA3, which encodes a protein with a shorter SR domain. Therefore, the level of atSRp34 protein was down-regulated, as the shorter version of the atSRp34 protein accumulated in plants [60]. A loss-of-function mutant of SR45, an SR-like protein, showed reduced root and shoot growth and multiple developmental defects and delayed flowering $[62,63]$. This mutant also exhibited hypersensitivity to glucose (Glc) and abscisic acid (ABA) during early seedling development [64] resistance to plant pathogens [65]. Regulation of glucose signaling by SR45 is accomplished through the modulation of degradation of the energy sensor SNF1-related kinase 1 (SnRNK1) in response to sugars [66]. AS of SR45 pre-mRNA produces two splice variants, SR45.1 (long isoform) and SR45.2 (short isoform), which encode proteins that differ in eight amino acids. Complementation of sr45 with 211 flower development, SR45.2 plays a role in root growth [63]. However, in terms of Glc and ABA 212 responses, both isoforms are able to complement the mutant phenotypes [64], suggesting distinct 213 and overlapping functions for SR45 splice isoforms. 
Despite the prevalence of alternative splicing of pre-mRNAs in plants, little is known about 216 the functions of splice isoforms generated from a gene. One of the reasons for this paucity is that 217 there are no simple, robust and easy to use methods to address splice isoform functions. Here we 218 describe three approaches based on emerging technologies that are likely to elucidate functions of 219 splice isoforms in plants.

i) CRISPR/Cas9-mediated genome engineering for functional analysis of SR proteins and their isoforms.

Recent advances in CRISPR/Cas9-mediated plant genome engineering make it possible to generate single and multiple functional knock-out mutants in diverse plant species (Figure 3A) [67-70]. For example, it will be possible to study the function of SR proteins in crop species due to the unprecedented efficiency of the CRISPR/Cas9 system in many of these species [71]. This simple and easy system involves the engineering of single guide-RNA molecules capable of targeting single or multiple genomic targets and generating functional knockouts and different protein variants. Using the CRISPR/Cas9 system, it is possible to insert or remove splice sites at will (see Figure 3 legend for details). Therefore, the contribution of certain splice variants could be studied by controlling the levels at which each isoform is made. Additionally, constitutive and alternative splice sites can be specifically modified, thereby allowing the study of the effects of these sequences on splicing and splice isoform levels. Recently Kang et al., 2018 [72] have used an adenine base editor fused to dCas9 to edit a $3^{\prime}$ acceptor site to promote mis-splicing of phytoene desaturase (PDS3) pre-mRNA. Such studies may lead to the engineering of plants with splice variants that will improve their performance under adverse stress conditions.

ii) Analysis of SR splice isoform functions by targeted degradation of specific splice variants using the CRISPR/Cas13 system utilized for RNA engineering, such as RNA interference, control of viral infections, RNA editing, and to increase transcriptome plasticity [73, 74]. Cas13a contains nucleotide-binding RNase domains that mediate target RNA cleavage. We recently determined the catalytic activity of CRISPR/Cas13a in plants, where we demonstrated interference against an RNA virus, Turnip 
Mosaic Virus [75]. Using the LwaCas13a system, mRNA levels of specific genes in rice

246 protoplasts could be reduced by over 50\% [76]. Although the targeted interference was modest,

247 Cas13a has many variants (Cas13b, Cas13d), some of which have enhanced activity in mammalian

248 cells [76-78] and could be further utilized in other eukaryotic systems, including plants.

As discussed earlier, the pre-mRNAs of SR proteins themselves undergo alternative splicing and produce multiple isoforms. Each of these isoforms has the potential to be expressed under specific stress and developmental conditions. It is important to study the effect of each splice isoform of an SR protein on plant growth and development and in stress responses. One possible way to address the function of individual splice variants is to target and degrade one specific isoform without affecting other splice variants. This can be achieved by CRISPR/Cas13a or other variants of Cas $13[77,78]$ in which a crRNA is designed to target a specific isoform (Figure 3B). We can use a crRNA against an intron sequence to target intron retention events under certain growth and developmental conditions (Figure 3B). Extensive research efforts are underway to use CRISPR/Cas13 systems for various RNA engineering applications. The CRISPR/Cas13 system can also be used for manipulation of alternative splicing by fusing the dCas13 (dead Cas13) to a particular SR protein [74]. In this case, CRISPR/Cas13 is used as an RNA-targeting complex carrying a functional domain (SR protein) to effect specific splicing outcomes (Figure 3C). Although alteration of specific splice isoforms using the CRISPR/cas13 system is yet to be demonstrated in plants, recently Konermann et al., 2018 [78] in a proof-of-concept study, have demonstrated that dCas13d can be used for targeted perturbation of splice isoform.

\section{iii) Identification of targets of SR proteins and their isoforms} understanding of SR proteins and their isoforms. However, global analysis of RNA targets of SR proteins in plants has been challenging due to limitations associated with widely used tools. Recently, key technologies have been developed that will advance the study of AS in plants, specifically in regard to SR proteins. For example, it is now possible to determine the targets of 273 immunoprecipitation sequencing (RIP-seq) (Figure 4A). In this method, RNAs bound to a specific 274 RBP are immunoprecipitated using a specific antibody [79] The RNA from immunoprecipitation 
$276[80,81]$. The use of antibodies requires the generation of specific high-quality antibodies for each 277 SR protein. This poses a problem as SRs in plants are encoded by gene families and also many 278 subfamilies of RBPs share considerable sequence similarity at the amino acid level in the RNA279 binding domain and other domains, leading to cross-reactivity of antibodies with other members 280 of the RBP family. To circumvent this problem, SR proteins fused to a tag (GFP or other tags) are expressed in a mutant background and antibodies to the tag are used for RIP. Other problems associated with RIP are the loss of true RNAs due to weak/unstable interactions. Post-lysis in vitro association of RBPs with non-specific RNA targets is also an issue in RIP-seq. Furthermore, RIPseq does not provide binding site information. RIP-seq has recently been used in Arabidopsis seedlings [82] and inflorescences [65] and the RNA associated with the SR45 (SARs) have been isolated and functionally annotated. About 4000 and 18000 transcripts associated with SR45 have been identified in seedlings and inflorescences, respectively. Gene Ontology (GO) analysis revealed them to be enriched for hormone and stress signaling pathways [82]

CLIP-seq, crosslinking immunoprecipitation followed by sequencing, is a modification of RIP-seq where an RBP is crosslinked with its bound cognate RNA by exposure to ultraviolet (UV) light $(254 \mathrm{~nm})$, which creates covalent links between protein and RNA [83]. An RBP-specific antibody is required to precipitate RNA bound to RBP and further treated with RNase to degrade non-bound RNAs. The extracted RNA is used for library preparation and high-throughput sequencing (Figure 4A). iCLIP (individual nucleotide resolution crosslinking immunoprecipitation) [84] and PAR-CLIP [85] are variants of CLIP. CLIP and iCLIP differ in library preparation methods whereas in PAR-CLIP cells/tissues are labeled with 4-thiouridine prior to UV $(365 \mathrm{~nm})$ crosslinking. With PAR-CLIP UV cross-linking efficiency is increased. The advantages of CLIP and its variants is that the RNA-RBP interactions are stabilized, hence stringent washing conditions can be used to remove spurious targets. Furthermore, these CLIPbased methods provide the binding site sequence information in target RNAs. As in RIP-seq, all CLIP methods require RBP-specific antibodies [86-88]. Also, the UV cross-linking is not very efficient but can also permanently capture transient interactions, which are difficult to separate at a later stage from biologically relevant interactions. False positives is another issue, as transcripts unaffected by RBP expression are identified as targets. CLIP is still technically challenging as the choice of RNase and fragmentation conditions can greatly affect target detection. Although CLIP and its variants have been widely used in animals, it has not been used to identify targets of any 
plant SR or SR-like proteins. So far, CLIP and iCLIP have been used to identify RNA targets of only two RBPs [89] in plants [90].

To overcome many of the issues with RIP and CLIP, an RNA tagging approach has recently been developed to probe protein-RNA interactions in vivo [91] (Figure 4B). In this method, an RBP is fused to the C. elegans poly (U) polymerase, PUP-2 [92]. Once the RBP binds an RNA in vivo, PUP leaves a covalent mark on the RNA, which is then detected by high-throughput sequencing. These covalent marks are a varied number of uridines (U-tag) on the $3^{\prime}$ end of the target RNAs. Before sequencing a reverse-transcription step is needed for library preparation that is selective for uridylated RNAs. However, as RNA tagging recognizes RNAs by their $\mathrm{U}$ tails it cannot identify the region bound by the RBP. Very recently another method was developed to identify RBP targets called TRIBE ( $\underline{\text { Targets of }}$ RNA-binding proteins Identified $\underline{B}$ E Editing) [9397] (Figure 4B). In its simplest application, an RBP is fused to the catalytic domain of the RNA-

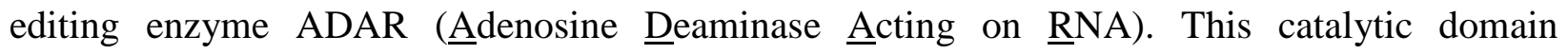
deaminases adenosine to inosine (A to I) [98]. Inosine is recognized as guanosine (G) both in vitro and in vivo. Once the RBP interacts with the target RNA, the RBP-fused ADAR catalytic domain irreversibly converts A to I and permanently marks the RNA. Subsequently, RNA is extracted and sequenced using high-throughput sequencing. The editing of A to $I(G)$ identifies the RNA targets. In addition, TRIBE can be performed with a small number of cells by expressing RBP-ADAR fusion protein using cell/tissue-specific promoter, hence capable of identifying cell/tissue-specific RNA targets. Disadvantages of this method are that it can't identify binding sites on RNA targets and the editing efficiency is low. A slightly modified version of TRIBE is HyperTRIBE in which the editing enzyme (ADAR) used has a single point mutation (E488Q) in the catalytic domain, which confers enhanced editing activity and significantly increases the sensitivity without sacrificing specificity [97].

\section{Future outlook} distinct structural features in plant-specific subfamilies. Understanding the molecular basis of their regulation with special emphasis on the plant-specific subfamilies remains an important subject for future research. Overall, dissecting the detailed molecular functions of the SR proteins in plants 
the function of single and multiple SR proteins. The emerging technologies described above will make it possible to generate functional knockouts of single and multiple genes of each plantspecific subfamily and determine the RNA targets and their regulation (Figure 5). The SR-target RNAs can be identified by RBP-dependent editing of target RNAs or TRIBE. For this purpose, the SR proteins will be fused with PUP or ADAR domains and expressed in plants. The targeted RNAs will be identified by high-throughput RNA sequencing. This information is the key to enhancing our understanding of the molecular roles of SR proteins in splicing regulation in response to various cellular and environmental cues. Subsequently, detailed molecular and physiological phenotyping will reveal the molecular roles of SR proteins in plant stress responses, growth, and development. Using these emerging technologies, it will be possible to tease apart the molecular function of single and multiple SR proteins and their splice isoforms in crop species. These studies will enhance our understanding of the molecular basis of plant adaptation to adverse environmental conditions and may help engineer plants with improved tolerance to biotic or abiotic stresses.

\section{Acknowledgements}

The study was supported by King Abdullah University of Science and Technology.

\section{Competing interests}

356 The authors declare no competing financial interests.

\section{References}

[1] X.D. Fu, The Superfamily of Arginine Serine-Rich Splicing Factors, Rna, 1 (1995) 663-680.

[2] P.J. Shepard, K.J. Hertel, The SR protein family, Genome Biol, 10 (2009) 242.

[3] A. Barta, M. Kalyna, A.S.N. Reddy, Implementing a rational and consistent nomenclature for serine/arginine-rich protein splicing factors (SR proteins) in plants, Plant Cell, 22 (2010) 29262929.

[4] D.N. Richardson, M.F. Rogers, A. Labadorf, A. Ben-Hur, H. Guo, A.H. Paterson, A.S. Reddy, Comparative analysis of serine/arginine-rich proteins across 27 eukaryotes: insights into subfamily classification and extent of alternative splicing, Plos One, 6 (2011) e24542.

[5] P. Duque, A role for SR proteins in plant stress responses, Plant Signal Behav, 6 (2011) 4954. 
[6] H.B. Rauch, T.L. Patrick, K.M. Klusman, F.U. Battistuzzi, W. Mei, V.P. Brendel, S.K. Lal, Discovery and expression analysis of alternative splicing events conserved among plant SR proteins, Mol Biol Evol, 31 (2014) 605-613.

[7] Q. Yan, X. Xia, Z. Sun, Y. Fang, Depletion of Arabidopsis SC35 and SC35-like serine/arginine-rich proteins affects the transcription and splicing of a subset of genes, PLoS Genet, 13 (2017) e1006663.

[8] G. Lazar, T. Schaal, T. Maniatis, H.M. Goodman, Identification of a Plant Serine-ArginineRich Protein Similar to the Mammalian Splicing Factor Sf2/Asf, Proc Natl Acad Sci U S A, 92 (1995) 7672-7676.

[9] S. Lopato, A. Mayeda, A.R. Krainer, A. Barta, Pre-mRNA splicing in plants: Characterization of Ser/Arg splicing factors, Proc Natl Acad Sci U S A, 93 (1996) 3074-3079.

[10] A. Barta, K. Sommergruber, D. Thompson, K. Hartmuth, M.A. Matzke, A.J. Matzke, The expression of a nopaline synthase - human growth hormone chimaeric gene in transformed tobacco and sunflower callus tissue, Plant Mol Biol, 6 (1986) 347-357.

[11] V.L. van Santen, R.A. Spritz, Splicing of plant pre-mRNAs in animal systems and vice versa, Gene, 56 (1987) 253-265.

[12] A.R. Kornblihtt, I.E. Schor, M. Allo, G. Dujardin, E. Petrillo, M.J. Munoz, Alternative splicing: a pivotal step between eukaryotic transcription and translation (vol 14, pg 153, 2013), Nat Rev Mol Cell Biol, 14 (2013).

[13] N.H. Syed, M. Kalyna, Y. Marquez, A. Barta, J.W.S. Brown, Alternative splicing in plants coming of age, Trends Plant Sci, 17 (2012) 616-623.

[14] G. Zhang, G. Guo, X. Hu, Y. Zhang, Q. Li, R. Li, R. Zhuang, Z. Lu, Z. He, X. Fang, L. Chen, W. Tian, Y. Tao, K. Kristiansen, X. Zhang, S. Li, H. Yang, J. Wang, J. Wang, Deep RNA sequencing at single base-pair resolution reveals high complexity of the rice transcriptome, Genome Res, 20 (2010) 646-654.

[15] M. Aouida, A. Eid, M.M. Mahfouz, CRISPR/Cas9-mediated target validation of the splicing inhibitor Pladienolide B, Biochim Open, 3 (2016) 72-75.

[16] B.B. Wang, V. Brendel, Genomewide comparative analysis of alternative splicing in plants, Proc Natl Acad Sci U S A, 103 (2006) 7175-7180.

[17] E.T. Wang, R. Sandberg, S. Luo, I. Khrebtukova, L. Zhang, C. Mayr, S.F. Kingsmore, G.P. Schroth, C.B. Burge, Alternative isoform regulation in human tissue transcriptomes, Nature, 456 (2008) 470-476.

[18] Y. Marquez, J.W.S. Brown, C. Simpson, A. Barta, M. Kalyna, Transcriptome survey reveals increased complexity of the alternative splicing landscape in Arabidopsis, Genome Res, 22 (2012) 1184-1195.

[19] Y. Ling, S. Alshareef, H. Butt, J. Lozano-Juste, L. Li, A.A. Galal, A. Moustafa, A.A. Momin, M. Tashkandi, D.N. Richardson, H. Fujii, S. Arold, P.L. Rodriguez, P. Duque, M.M. Mahfouz, Pre-mRNA splicing repression triggers abiotic stress signaling in plants, Plant J, 89 (2017) 291309.

[20] S. AlShareef, Y. Ling, H. Butt, K.G. Mariappan, M. Benhamed, M.M. Mahfouz, Herboxidiene triggers splicing repression and abiotic stress responses in plants, BMC Genomics, 18 (2017) 260.

[21] S. Kervestin, A. Jacobson, NMD: a multifaceted response to premature translational termination, Nat Rev Mol Cell Biol, 13 (2012) 700-712.

[22] T. Laloum, G. Martin, P. Duque, Alternative Splicing Control of Abiotic Stress Responses, Trends Plant Sci, 23 (2018) 140-150.

[23] G.S. Ali, A.S.N. Reddy, Spatiotemporal organization of pre-mRNA splicing proteins in plants, in: Curr Top Microbiol Immunol, 2008, pp. 103-118.

[24] F.C. Chen, S.S. Wang, S.M. Chaw, Y.T. Huang, T.J. Chuang, Plant gene and alternatively spliced variant annotator. A plant genome annotation pipeline for rice gene and alternatively 
spliced variant identification with cross-species expressed sequence tag conservation from seven plant species, Plant Physiol, 143 (2007) 1086-1095.

[25] S.A. Filichkin, H.D. Priest, S.A. Givan, R. Shen, D.W. Bryant, S.E. Fox, W.K. Wong, T.C. Mockler, Genome-wide mapping of alternative splicing in Arabidopsis thaliana, Genome Res, 20 (2010) 45-58.

[26] F. Ding, P. Cui, Z.Y. Wang, S.D. Zhang, S. Ali, L.M. Xiong, Genome-wide analysis of alternative splicing of pre-mRNA under salt stress in Arabidopsis, BMC Genomics, 15 (2014). [27] Y. Ling, N. Serrano, G. Gao, M. Atia, M. Mokhtar, Y.H. Woo, J. Bazin, A. Veluchamy, M. Benhamed, M. Crespi, C. Gehring, A.S.N. Reddy, M.M. Mahfouz, Thermopriming triggers splicing memory in Arabidopsis, J Exp Bot, 69 (2018) 2659-2675.

[28] A.J. Matlin, F. Clark, C.W.J. Smith, Understanding alternative splicing: Towards a cellular code, Nat Rev Mol Cell Biol, 6 (2005) 386-398.

[29] L.A. Chasin, Searching for splicing motifs, Adv Exp Med Biol, 623 (2007) 85-106. [30] A.G. Matera, Z.F. Wang, A day in the life of the spliceosome, Nat Rev Mol Cell Bio, 15 (2014) 108-121.

[31] A. Barta, M. Kalyna, Z.J. Lorković, Plant SR proteins and their functions, in: Curr Top Microbiol Immunol, 2008, pp. 83-102.

[32] K.J. Hertel, B.R. Graveley, RS domains contact the pre-mRNA throughout spliceosome assembly, Trends Biochem Sci, 30 (2005) 115-118.

[33] J.M. Howard, J.R. Sanford, The RNAissance family: SR proteins as multifaceted regulators of gene expression, Wiley Interdiscip Rev RNA, 6 (2015) 93-110.

[34] H.H. Shen, M.R. Green, A pathway of sequential arginine-serine-rich domain-splicing signal interactions during mammalian spliceosome assembly, Mol Cell, 16 (2004) 363-373.

[35] H.H. Shen, J.L.C. Kan, M.R. Green, Arginine-serine-rich domains bound at splicing enhancers contact the branchpoint to promote prespliceosome assembly, Mol Cell, 13 (2004) 367-376.

[36] Y. Huang, J.A. Steitz, SRprises along a messenger's journey, Mol Cell, 17 (2005) 613-615. [37] A. Kalsotra, T.A. Cooper, Functional consequences of developmentally regulated alternative splicing, Nat Rev Genet, 12 (2011) 715-729.

[38] J.C. Long, J.F. Caceres, The SR protein family of splicing factors: Master regulators of gene expression, Biochem J, 417 (2009) 15-27.

[39] R. Reed, H. Cheng, TREX, SR proteins and export of mRNA, Curr Opin Cell Biol, 17 (2005) 269-273.

[40] Y.Q. Huang, T.A. Yario, J.A. Steitz, A molecular link between SR protein dephosphorylation and mRNA export, Proc Natl Acad Sci U S A, 101 (2004) 9666-9670.

[41] X.Y. Zhong, J.H. Ding, J.A. Adams, G. Ghosh, X.D. Fu, Regulation of SR protein phosphorylation and alternative splicing by modulating kinetic interactions of SRPK1 with molecular chaperones, Gene Dev, 23 (2009) 482-495.

[42] S. Xu, Z. Zhang, B. Jing, P. Gannon, J. Ding, F. Xu, X. Li, Y. Zhang, Transportin-SR is required for proper splicing of resistance genes and plant immunity, PLoS Genet, 7 (2011) e1002159.

[43] B.R. Graveley, Sorting out the complexity of SR protein functions, RNA, 6 (2000) 11971211.

[44] S.G. Palusa, A.S.N. Reddy, Extensive coupling of alternative splicing of pre-mRNAs of serine/arginine (SR) genes with nonsense-mediated decay, New Phytol, 185 (2010) 83-89.

[45] N. Tanabe, K. Yoshimura, A. Kimura, Y. Yabuta, S. Shigeoka, Differential expression of alternatively spliced mRNAs of Arabidopsis SR protein homologs, atSR30 and atSR45a, in response to environmental stress, Plant Cell Physiol, 48 (2007) 1036-1049.

[46] K. lida, M. Go, Survey of conserved alternative splicing events of mRNAs encoding SR proteins in land plants, Mol Biol Evol, 23 (2006) 1085-1094. 
[47] M. Kalyna, S. Lopato, V. Voronin, A. Barta, Evolutionary conservation and regulation of particular alternative splicing events in plant SR proteins, Nucleic Acids Res, 34 (2006) 43954405.

[48] Z. Zhou, X.D. Fu, Regulation of splicing by SR proteins and SR protein-specific kinases, Chromosoma, 122 (2013) 191-207.

[49] S. Savaldi-Goldstein, G. Sessa, R. Fluhr, The ethylene-inducible PK12 kinase mediates the phosphorylation of SR splicing factors, Plant J, 21 (2000) 91-96.

[50] B.B. Wang, V. Brendel, The ASRG database: identification and survey of Arabidopsis thaliana genes involved in pre-mRNA splicing, Genome Biol, 5 (2004).

[51] M. Golovkin, A.S.N. Reddy, An SC35-like protein and a novel serine/arginine-rich protein interact with Arabidopsis U1-70K protein, J Biol Chem, 274 (1999) 36428-36438.

[52] S.G. Palusa, G.S. Ali, A.S.N. Reddy, Alternative splicing of pre-mRNAs of Arabidopsis serine/arginine-rich proteins: Regulation by hormones and stresses, Plant J, 49 (2007) 10911107.

[53] G.S. Ali, M. Golovkin, A.S.N. Reddy, Nuclear localization and in vivo dynamics of a plantspecific serine/arginine-rich protein, Plant J, 36 (2003) 883-893.

[54] G. Rausin, V. Tillemans, N. Stankovic, M. Hanikenne, P. Motte, Dynamic

Nucleocytoplasmic Shuttling of an Arabidopsis SR Splicing Factor: Role of the RNA-Binding Domains, Plant Physiol, 153 (2010) 273-284.

[55] V. Tillemans, I. Leponce, G. Rausin, L. Dispa, P. Motte, Insights into nuclear organization in plants as revealed by the dynamic distribution of Arabidopsis SR splicing factors, Plant Cell, 18 (2006) 3218-3234.

[56] G.S. Ali, A.S. Reddy, ATP, phosphorylation and transcription regulate the mobility of plant splicing factors, J Cell Sci, 119 (2006) 3527-3538.

[57] S.P. Dinesh-Kumar, B.J. Baker, Alternatively spliced N resistance gene transcripts: their possible role in tobacco mosaic virus resistance, Proc Natl Acad Sci U S A, 97 (2000) 19081913.

[58] C. Egawa, F. Kobayashi, M. Ishibashi, T. Nakamura, C. Nakamura, S. Takumi, Differential regulation of transcript accumulation and alternative splicing of a DREB2 homolog under abiotic stress conditions in common wheat, Genes Genet Syst, 81 (2006) 77-91.

[59] C. Dong, F. He, O. Berkowitz, J. Liu, P. Cao, M. Tang, H. Shi, W. Wang, Q. Li, Z. Shen, J. Whelan, L. Zheng, Alternative Splicing Plays a Critical Role in Maintaining Mineral Nutrient Homeostasis in Rice (Oryza sativa), Plant Cell, 30 (2018) 2267-2285.

[60] S. Lopato, M. Kalyna, S. Dorner, R. Kobayashi, A.R. Krainer, A. Barta, atSRp30, one of two SF2/ASF-like proteins from Arabidopsis thaliana, regulates splicing of specific plant genes, Genes Dev, 13 (1999) 987-1001.

[61] M. Kalyna, S. Lopato, A. Barta, Ectopic expression of atRSZ33 reveals its function in splicing and causes pleiotropic changes in development, Mol Biol Cell, 14 (2003) 3565-3577. [62] G.S. Ali, S.G. Palusa, M. Golovkin, J. Prasad, J.L. Manley, A.S.N. Reddy, Regulation of Plant Developmental Processes by a Novel Splicing Factor, Plos One, 2 (2007).

[63] X.N. Zhang, S.M. Mount, Two alternatively spliced isoforms of the Arabidopsis SR45 protein have distinct roles during normal plant development, Plant Physiol, 150 (2009) 14501458.

[64] R.F. Carvalho, S.D. Carvalho, P. Duque, The plant-specific SR45 protein negatively regulates glucose and ABA signaling during early seedling development in Arabidopsis, Plant Physiol, 154 (2010) 772-783.

[65] X.N. Zhang, Y. Shi, J.J. Powers, N.B. Gowda, C. Zhang, H.M.M. Ibrahim, H.B. Ball, S.L. Chen, H. Lu, S.M. Mount, Transcriptome analyses reveal SR45 to be a neutral splicing regulator and a suppressor of innate immunity in Arabidopsis thaliana, BMC Genomics, 18 (2017) 772. 
[66] R.F. Carvalho, D. Szakonyi, C.G. Simpson, I.C. Barbosa, J.W. Brown, E. Baena-Gonzalez, P. Duque, The Arabidopsis SR45 Splicing Factor, a Negative Regulator of Sugar Signaling, Modulates SNF1-Related Protein Kinase 1 Stability, Plant Cell, 28 (2016) 1910-1925. [67] A. Piatek, M.M. Mahfouz, Targeted genome regulation via synthetic programmable transcriptional regulators, Crit Rev Biotechnol, (2016) 1-12.

[68] M. Tashkandi, Z. Ali, F. Aljedaani, A. Shami, M.M. Mahfouz, Engineering resistance against Tomato yellow leaf curl virus via the CRISPR/Cas9 system in tomato, Plant Signal Behav, 13 (2018) e1525996.

[69] H. Butt, M. Jamil, J.Y. Wang, S. Al-Babili, M. Mahfouz, Engineering plant architecture via CRISPR/Cas9-mediated alteration of strigolactone biosynthesis, BMC Plant Biol, 18 (2018) 174. [70] V. Nekrasov, B. Staskawicz, D. Weigel, J.D. Jones, S. Kamoun, Targeted mutagenesis in the model plant Nicotiana benthamiana using Cas9 RNA-guided endonuclease, Nat Biotechnol, 31 (2013) 691-693.

[71] C. Gao, The future of CRISPR technologies in agriculture, Nat Rev Mol Cell Biol, 19 (2018) 275-276.

[72] B.C. Kang, J.Y. Yun, S.T. Kim, Y. Shin, J. Ryu, M. Choi, J.W. Woo, J.S. Kim, Precision genome engineering through adenine base editing in plants, Nat Plants, 4 (2018) 427-431. [73] Z. Ali, A. Mahas, M. Mahfouz, CRISPR/Cas13 as a Tool for RNA Interference, Trends Plant Sci, 23 (2018) 374-378.

[74] A. Mahas, C. Neal Stewart, Jr., M.M. Mahfouz, Harnessing CRISPR/Cas systems for programmable transcriptional and post-transcriptional regulation, Biotechnol Adv, 36 (2018) 295-310.

[75] R. Aman, Z. Ali, H. Butt, A. Mahas, F. Aljedaani, M.Z. Khan, S. Ding, M. Mahfouz, RNA virus interference via CRISPR/Cas13a system in plants, Genome Biol, 19 (2018) 1.

[76] O.O. Abudayyeh, J.S. Gootenberg, P. Essletzbichler, S. Han, J. Joung, J.J. Belanto, V. Verdine, D.B.T. Cox, M.J. Kellner, A. Regev, E.S. Lander, D.F. Voytas, A.Y. Ting, F. Zhang, RNA targeting with CRISPR-Cas13, Nature, 550 (2017) 280-284.

[77] D.B.T. Cox, J.S. Gootenberg, O.O. Abudayyeh, B. Franklin, M.J. Kellner, J. Joung, F. Zhang, RNA editing with CRISPR-Cas13, Science, 358 (2017) 1019-1027.

[78] S. Konermann, P. Lotfy, N.J. Brideau, J. Oki, M.N. Shokhirev, P.D. Hsu, Transcriptome Engineering with RNA-Targeting Type VI-D CRISPR Effectors, Cell, 173 (2018) 665-676 e614. [79] E.C. Wheeler, E.L. Van Nostrand, G.W. Yeo, Advances and challenges in the detection of transcriptome-wide protein-RNA interactions, Wiley Interdiscip Rev RNA, 9 (2018). [80] S. Mili, J.A. Steitz, Evidence for reassociation of RNA-binding proteins after cell lysis: implications for the interpretation of immunoprecipitation analyses, RNA, 10 (2004) 1692-1694. [81] C.A. McHugh, P. Russell, M. Guttman, Methods for comprehensive experimental identification of RNA-protein interactions, Genome Biol, 15 (2014) 203.

[82] D. Xing, Y. Wang, M. Hamilton, A. Ben-Hur, A.S. Reddy, Transcriptome-Wide Identification of RNA Targets of Arabidopsis SERINE/ARGININE-RICH45 Uncovers the Unexpected Roles of This RNA Binding Protein in RNA Processing, Plant Cell, 27 (2015) 3294-3308.

[83] J. Ule, K.B. Jensen, M. Ruggiu, A. Mele, A. Ule, R.B. Darnell, CLIP identifies Novaregulated RNA networks in the brain, Science, 302 (2003) 1212-1215.

[84] J. Konig, K. Zarnack, G. Rot, T. Curk, M. Kayikci, B. Zupan, D.J. Turner, N.M. Luscombe, J. Ule, iCLIP reveals the function of hnRNP particles in splicing at individual nucleotide resolution, Nat Struct Mol Biol, 17 (2010) 909-915.

[85] M. Hafner, M. Landthaler, L. Burger, M. Khorshid, J. Hausser, P. Berninger, A. Rothballer, M. Ascano, Jr., A.C. Jungkamp, M. Munschauer, A. Ulrich, G.S. Wardle, S. Dewell, M. Zavolan, T. Tuschl, Transcriptome-wide identification of RNA-binding protein and microRNA target sites by PAR-CLIP, Cell, 141 (2010) 129-141. 
[86] S. Kishore, L. Jaskiewicz, L. Burger, J. Hausser, M. Khorshid, M. Zavolan, A quantitative analysis of CLIP methods for identifying binding sites of RNA-binding proteins, Nat Methods, 8 (2011) 559-564.

[87] M.J. Moore, C. Zhang, E.C. Gantman, A. Mele, J.C. Darnell, R.B. Darnell, Mapping Argonaute and conventional RNA-binding protein interactions with RNA at single-nucleotide resolution using HITS-CLIP and CIMS analysis, Nat Protoc, 9 (2014) 263-293.

[88] E.L. Van Nostrand, G.A. Pratt, A.A. Shishkin, C. Gelboin-Burkhart, M.Y. Fang, B. Sundararaman, S.M. Blue, T.B. Nguyen, C. Surka, K. Elkins, R. Stanton, F. Rigo, M. Guttman, G.W. Yeo, Robust transcriptome-wide discovery of RNA-binding protein binding sites with enhanced CLIP (eCLIP), Nat Methods, 13 (2016) 508-514.

[89] K. Meyer, T. Koster, C. Nolte, C. Weinholdt, M. Lewinski, I. Grosse, D. Staiger, Adaptation of iCLIP to plants determines the binding landscape of the clock-regulated RNA-binding protein AtGRP7, Genome Biol, 18 (2017) 204.

[90] Y. Zhang, L. Gu, Y. Hou, L. Wang, X. Deng, R. Hang, D. Chen, X. Zhang, Y. Zhang, C. Liu, $X$. Cao, Integrative genome-wide analysis reveals HLP1, a novel RNA-binding protein, regulates plant flowering by targeting alternative polyadenylation, Cell Res, 25 (2015) 864-876.

[91] C.P. Lapointe, D. Wilinski, H.A. Saunders, M. Wickens, Protein-RNA networks revealed through covalent RNA marks, Nat Methods, 12 (2015) 1163-1170.

[92] C.P. Lapointe, M. Wickens, The nucleic acid-binding domain and translational repression activity of a Xenopus terminal uridylyl transferase, J Biol Chem, 288 (2013) 20723-20733.

[93] A.C. McMahon, R. Rahman, H. Jin, J.L. Shen, A. Fieldsend, W. Luo, M. Rosbash, TRIBE: Hijacking an RNA-Editing Enzyme to Identify Cell-Specific Targets of RNA-Binding Proteins, Cell, 165 (2016) 742-753.

[94] D.A. Burow, S. Martin, J.F. Quail, N. Alhusaini, J. Coller, M.D. Cleary, Attenuated Codon Optimality Contributes to Neural-Specific mRNA Decay in Drosophila, Cell Rep, 24 (2018) 17041712.

[95] P. Vogel, M. Moschref, Q. Li, T. Merkle, K.D. Selvasaravanan, J.B. Li, T. Stafforst, Efficient and precise editing of endogenous transcripts with SNAP-tagged ADARs, Nat Methods, 15 (2018) 535-538.

[96] G. Benegiamo, L.S. Mure, G. Erikson, H.D. Le, E. Moriggi, S.A. Brown, S. Panda, The RNA-Binding Protein NONO Coordinates Hepatic Adaptation to Feeding, Cell Metab, 27 (2018) 404-418 e407.

[97] R. Rahman, W. Xu, H. Jin, M. Rosbash, Identification of RNA-binding protein targets with HyperTRIBE, Nat Protoc, 13 (2018) 1829-1849.

[98] L.P. Keegan, A. Leroy, D. Sproul, M.A. O'Connell, Adenosine deaminases acting on RNA (ADARs): RNA-editing enzymes, Genome Biol, 5 (2004) 209. 


\section{Figure Legends}

\section{Figure 1}

615 Schematic representation of domain structures of the distinct SR protein subfamilies with members

616 in several plant species. A) Highly conserved eukaryotic SR protein subfamilies. B) Plant-specific

617 SR protein subfamilies.

\section{Figure 2}

619 A typical pre-mRNA sequence with exons (represented as boxes) and introns (represented as lines).

620 The cis-regulatoty elements are represented at the top including 5'splice site, branch point, 621 polypyrimidine tract, 3'splice site, ESS, ESE, ISS and ISE. These cis-elements are recognized by 622 trans-acting factors, which consist of small nuclear ribonucleoproteins (snRNPs) and numerous 623 additional proteins. Binding of SR proteins and hnRNPs (heterogeneous ribonucleoproteins) to 624 SREs and their effect on splicing (promotion/inhibition) is shown. The splicing reaction is 625 regulated by multiple environmental and developmental signals, which ultimately leads to various 626 splicing outcomes.

\section{$627 \quad$ Figure 3}

628 A) CRISPR/Cas9-mediated genome engineering. Recognition of target genomic DNA sequence 629 by Cas9 (CRISPR-associated protein 9) RNA-guided DNA endonuclease enzyme is mediated by 630 complementary binding to the guide RNA, guided by the detection of PAM (protospacer adjacent 631 motif) sequences. Cas9 then catalyzes the double-stranded cleavage of genomic DNA near the 632 PAM sequence, producing a double-stranded nick. The cleaved genomic DNA is then repaired, 633 either via the non-homologous end-joining pathway (NHEJ) that yields a genomic DNA containing 634 an indel, or via the homology-directed repair mechanism (HDR) which uses homologous donor 635 DNA to guide repair, leading to the introduction of a novel sequence into genomic DNA. Both 636 mechanisms can lead to a shift in the open reading frame of the target gene, frequently leading to 637 a premature stop codon and subsequent gene silencing. 
638 B) The CRISPR/Cas13 system is used for RNA targeting in eukaryotes. The spacer sequence 639 targets the mRNA near the PFS (protospacer flanking site). The spacer sequence can be designed 640 to target a specific isoform of SR mRNAs to identify the biological role of that isoform.

641 C) Pre-mRNA splicing could be altered by fusing dCas13a (dead Cas13a) with SR proteins. 642 dCas13a can be programmed to recruit an SR protein to a specific splicing regulatory signal, which 643 would allow manipulation of specific isoform levels.

\section{Figure 4}

645 A) In RIP-seq, after lysis of cells of interest, RNPs (ribonucleoproteins, i.e. RNA molecules bound 646 with RBPs) are extracted and purified before being immunoprecipitated using bead-bound 647 antibodies raised against the RBPs of interest. Bound RNA fragments are eluted and used to 648 prepare cDNA libraries, which are then sequenced to identify the binding targets of the RBPs of 649 interest. CLIP-seq has several modifications. The RBPs are covalently crosslinked to their bound 650 cognate RNAs using UV irradiation before cells are lysed and RNPs extracted and purified. The 651 resulting RNPs are immunoprecipitated using bead-bound antibodies raised against the RBPs of 652 interest. The resulting samples are subjected to RNase degradation that eliminates unbound RNAs 653 as well as fragments of RNAs that are not involved in RBP binding. After elution, the remaining 654 RNA sample, which is enriched in binding sites of the RBPs of interest, is used to make cDNA 655 libraries that are sequenced for identification and quantification.

656 B) For the in vivo identification of RNA targets, SR proteins are fused with other enzymes that add 657 a tag or marks an editing event. In RNA tagging, the SR protein is fused with the C. elegans PUP 658 (poly (U) polymerase) domain. The chimeric protein covalently adds several uridines (U-tag) to 659 the tail of RNAs bound with RBP, i.e. to SR. cDNA library preparation involves tagging of 660 modified $U$ tails with guanosines $(\mathrm{G})$ and inosines (I) and later RNA sequencing. In another method 661 known as TRIBE, the SR protein is fused with the catalytic domain of Drosophila ADAR. The 662 ADAR converts adenosine (A) to inosine (I) which is recognized as guanosine. The SR protein 663 RNA targets are marked by an irreversible editing event at the binding site which is later identified 664 by library preparation and RNA sequencing. 
667 An experimental platform for molecular and physiological phenotyping of SR proteins. The role 668 of SR proteins in determining tolerance and sensitivity to environmental stress can be achieved by 669 characterizing plant varieties subject to different stresses. 1) Single or multiple SR proteins within 670 given subfamilies can be knocked out using the CRISPR/Cas9 system. We can use the 671 CRISPR/Cas13 system to target SR protein transcripts for a specific RNA isoform or can fuse 672 dCas13 with SR protein to promote specific splicing event. 2) The resulting lines can then be 673 characterized at the molecular level using RNA-seq, and CLIP-seq to determine the variety674 specific effects of SR protein knockout in terms of AS profiles, SR protein targets and binding 675 sites. For more precise molecular analysis RNA tagging approach/ TRIBE method can be 676 employed. In parallel, these lines can be phenotyped to determine the effect of each mutant on 677 stress tolerance, measuring key traits such as biomass, growth, yield and survival. 3) Finally, 678 correlation analyses can be utilized to determine the role of each SR protein/subfamily in stress 679 tolerance. Genes shown to be alternatively spliced in an SR protein-dependent manner, with effects 680 on stress tolerance, can be subjected to further functional characterization. 


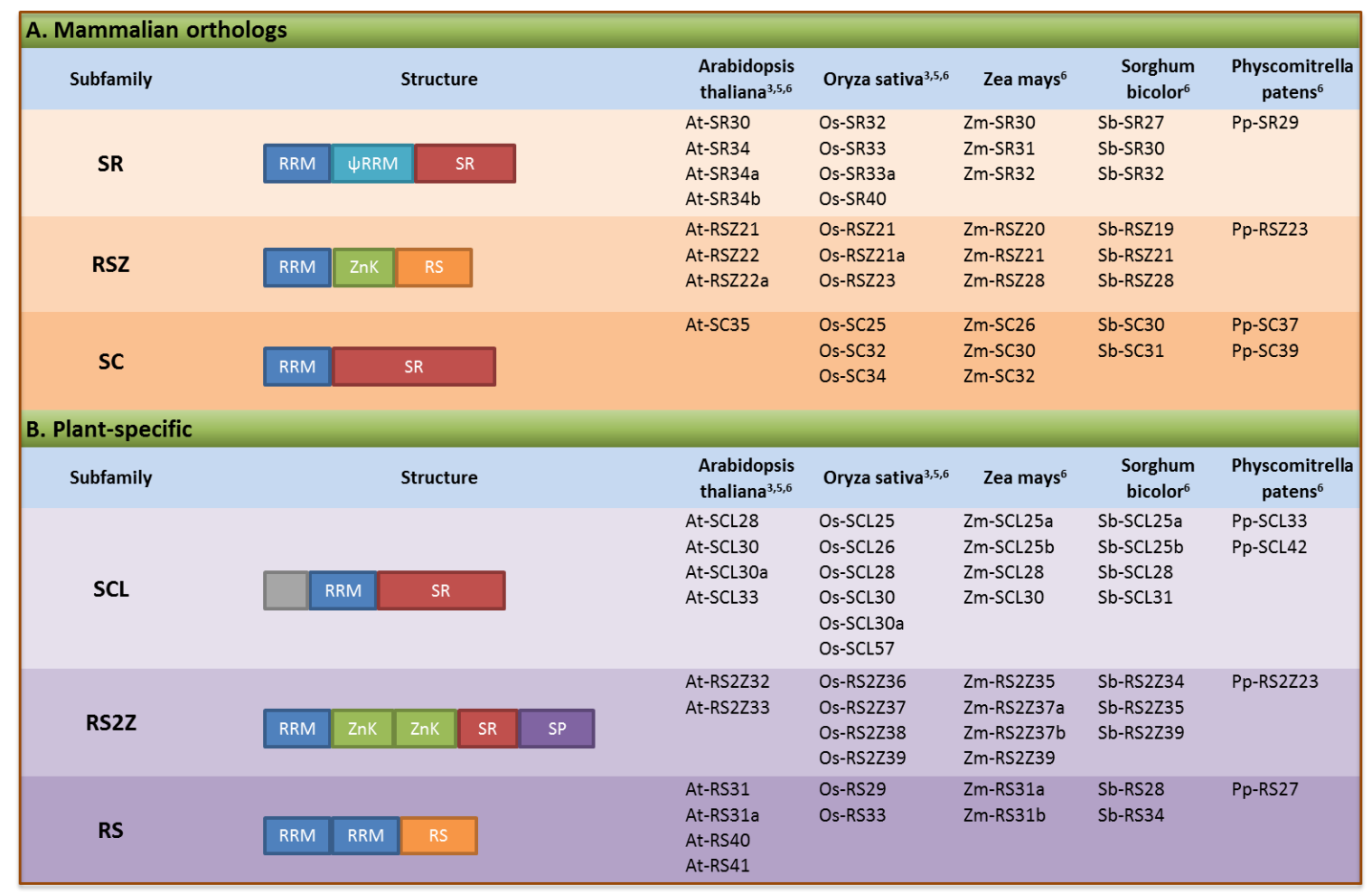

\begin{tabular}{|c|c|c|c|}
\hline RRM & RRM (RNA Recognition motif) domain & SP & (Serine-Proline (SP) repeat) \\
\hline URRM & RRMH (RRM Homology) domain, posses SWQDLKD motif & & Arg, Pro, Ser, Gly, Tyr rich extension \\
\hline SR & SR domain (Serine-Arginine (SR) repeat) & RS & RS domain (Arginine-Serine (RS) repeat) \\
\hline ZnK & Zinc binding domain & & \\
\hline
\end{tabular}

\section{$691 \quad$ Figure 1}




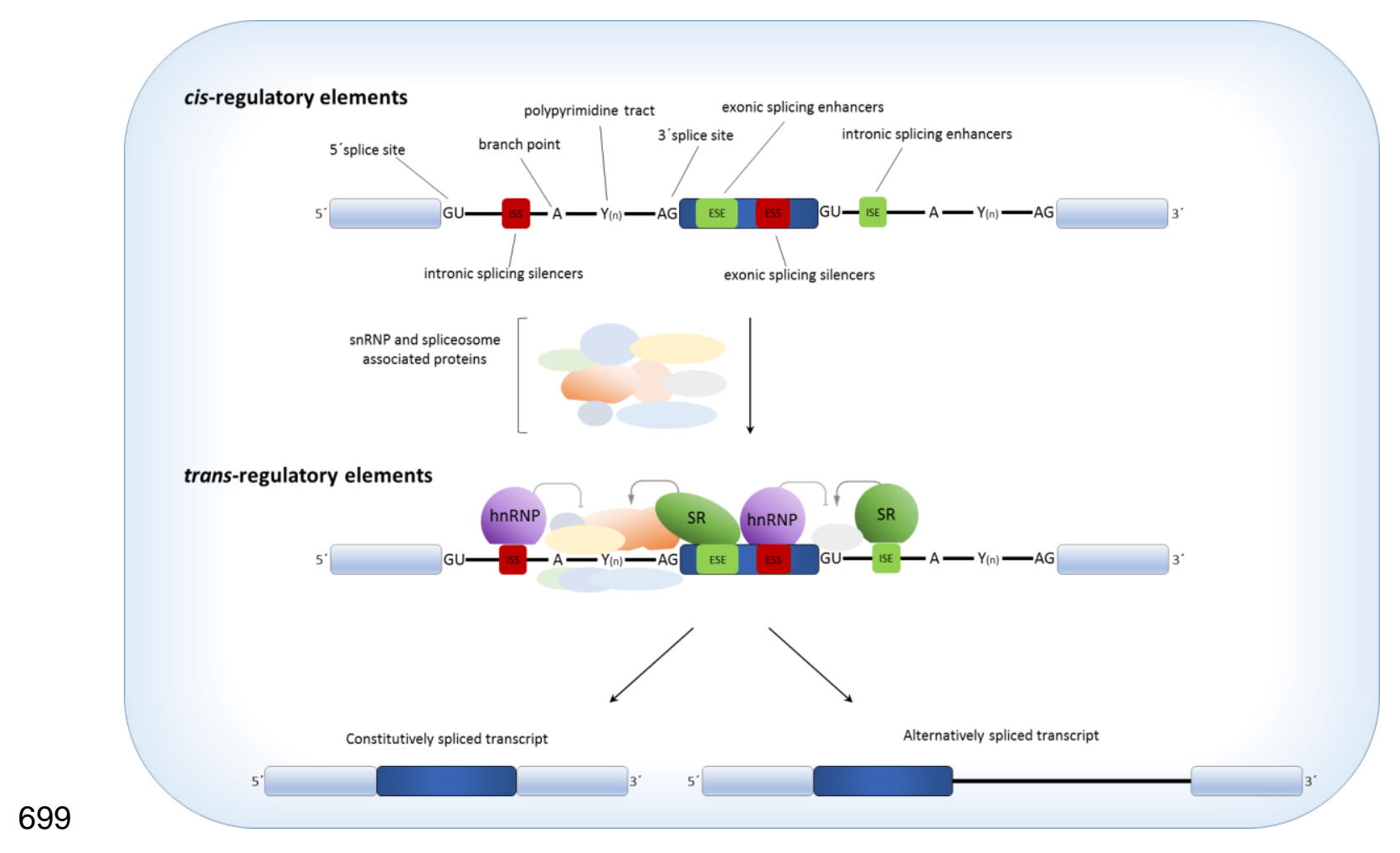

$700 \quad$ Figure 2

701

702

703

704

705

706

707

708

709 

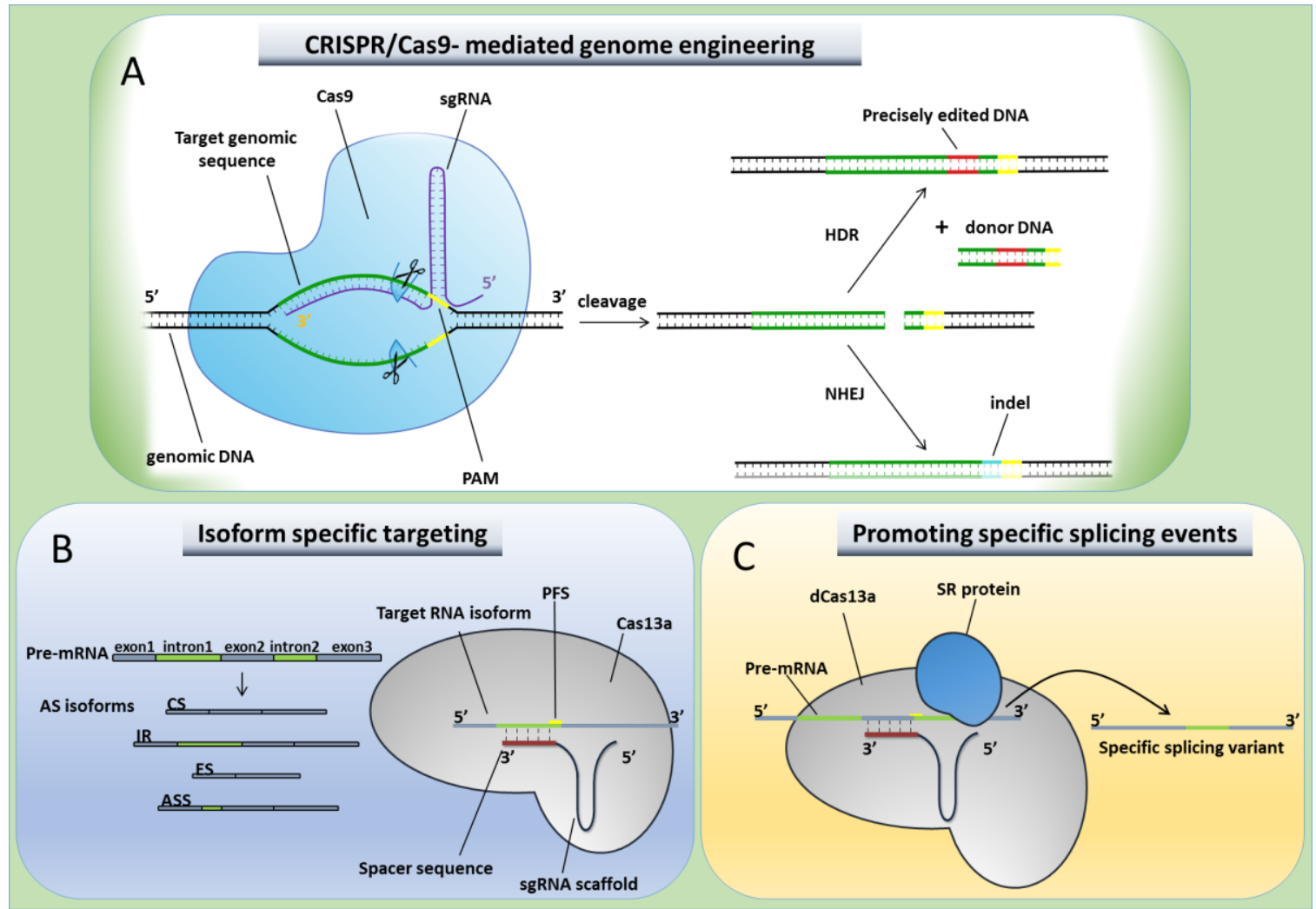

\section{$711 \quad$ Figure 3}




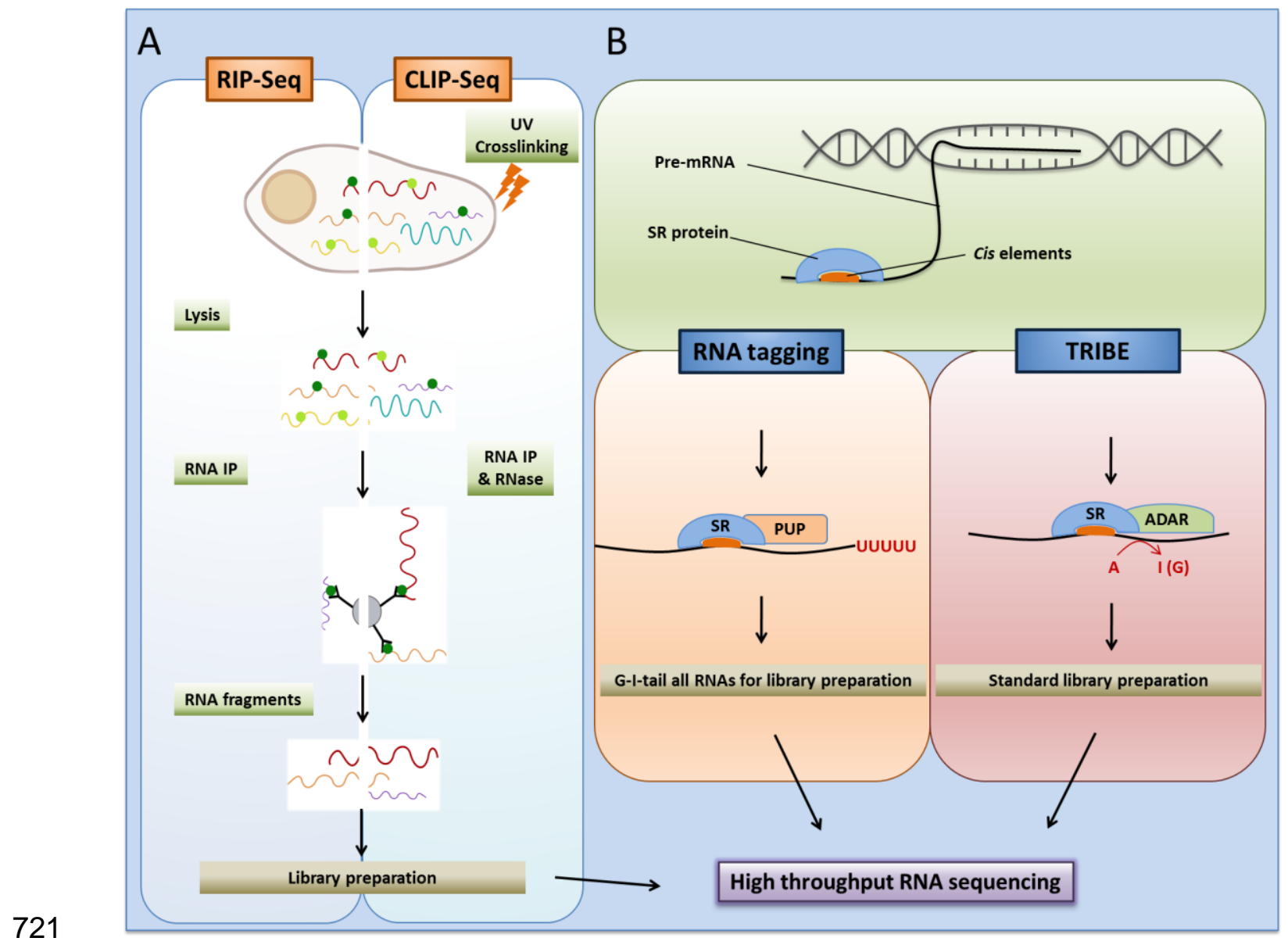

$722 \quad$ Figure 4

723

724

725

726

727

728

729 


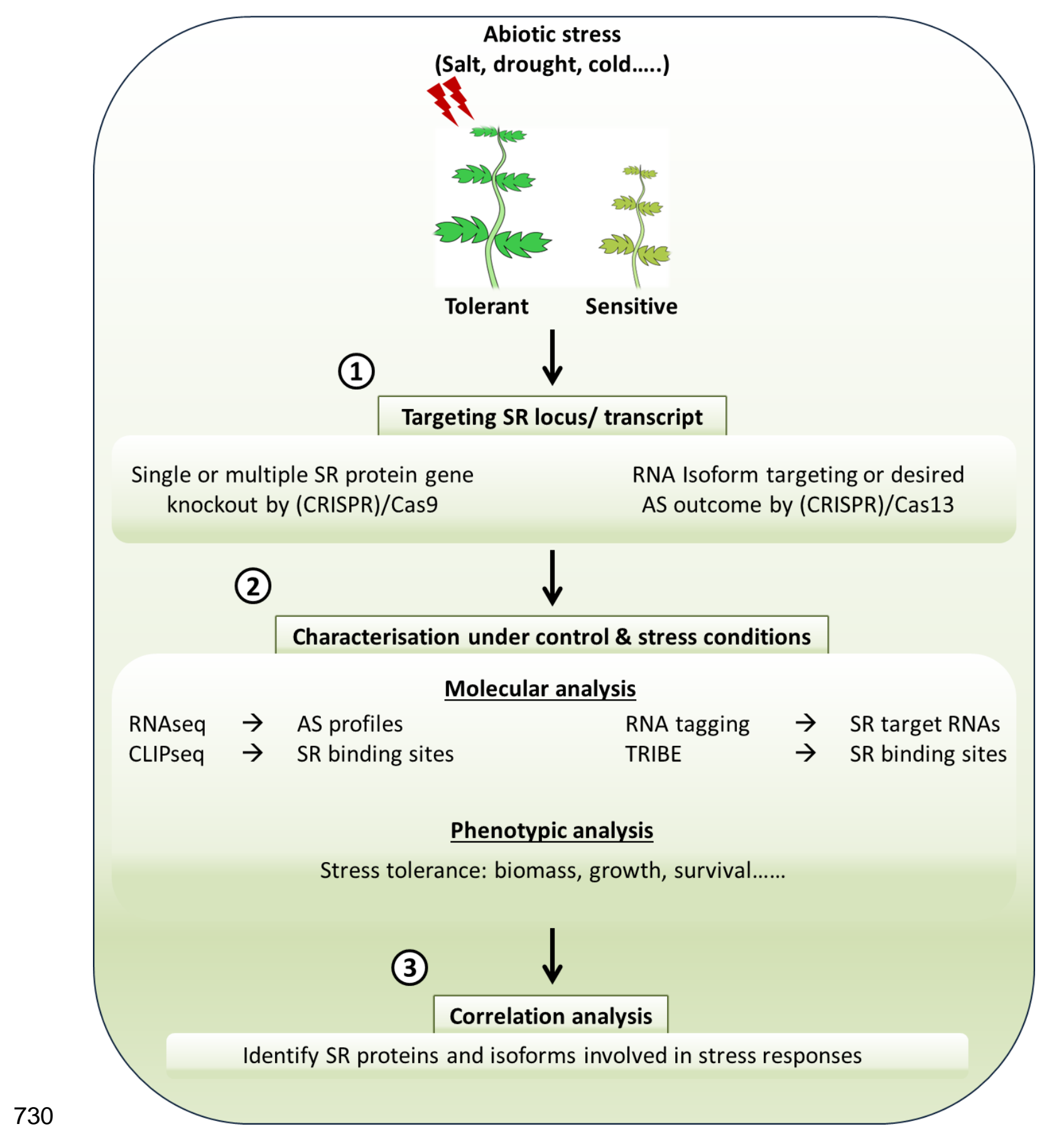

$731 \quad$ Figure 5 